\title{
GRAVITATIONAL LENSING AND COSMIC STRINGS
}

\author{
Rachel Webster \\ Department of Astronomy, University of Toronto. \\ Paul Hewett \\ Institute of Astronomy, Cambridge.
}

Cosmic strings would be cosmologically interesting if they acted as seeds for rich cluster and galaxy formation. Most comological scenarios require the mean mass density per unit length of the string $\mu$ to be $\sim 2 \times 10^{-6}$ for cosmic strings to act as seeds. If there is a population of long-lived cosmic strings which are either long loops or infinite strings, then they will gravitationally lense background sources (Vilenkin 1984). We are undertaking a substantial survey for multiply imaged optical quasars. So far we have examined $\sim 2500$ quasars and have no candidate multiply imaged quasars with separations $\gtrsim 13 "(W e b s t e r$ et al. 1987). This sample will double over the next year. The survey will detect multiply imaged quasars with the image separations predicted if one has a population of cosmic strings with $\mu \sim 2 \times 10^{-6}$ acting as gravitational lenses. In due course, this survey will enable us to confirm or rule out the possibility that cosmic strings seed galaxy formation.

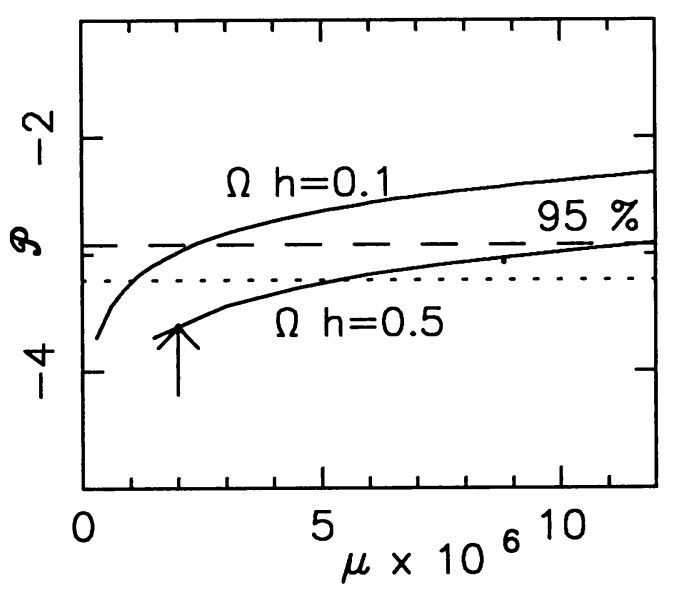

Figure 1. The solid lines show the plot of the logarithm of the probability $\mathcal{P}$ of detecting a double image against the mass per unit length of a cosmic string, $\mu$. Curves for two different values of $\Omega h$ are given, where $h$ is the Hubble constant in units of $100 \mathrm{~km} \mathrm{~s}^{-1} M p c^{-1}$. The dashed line gives the $95 \%$ confidence upper limit for the sample of quasars which has been examined so far. The dotted line shows the $95 \%$ confidence limit for a sample of $\sim 5000$ quasars. The arrow shows the value of $\mu$ required to seed galaxy formation in the Cold Dark Matter or neutrino dominated cosmological models.

\section{REFERENCES}

Vilenkin, A. 1984, Astrophys. J., 282, L51.

Webster, R.L., Hewett, P.C. and Irwin, M.J. 1987, Astron. J., , in press. 